\title{
Components of Phylogenetic Signal in Antagonistic and Mutualistic Networks
}

\author{
Rudolf P. Rohr* and Jordi Bascompte \\ Integrative Ecology Group, Estación Biológica de Doñana, Consejo Superior de Investigaciones Científicas, Calle Américo \\ Vespucio s/n, E-41092 Sevilla, Spain
}

\begin{abstract}
Recent studies have shown a phylogenetic signal in the structure of ecological networks, making the point that evolutionary history is important in explaining network architecture. However, this previous work has focused on either antagonistic (i.e., predatorprey) or mutualistic networks and has used different methodologies. Thus, a comparative assessment of both the frequency and the strength of phylogenetic signal across network types and components of network structure has been precluded. Here, we address this issue using a data set comprising 60 antagonistic and mutualistic networks. By quantifying simultaneously the matching and centrality components of network architecture-capturing the modular and nested structure, respectively-we test the presence and quantify the strength of phylogenetic signal across network types, species sets, and components of network structure. We find contrasting differences across such groups. First, phylogenetic signal is stronger in antagonistic webs than in mutualistic webs. Second, resources are more strongly constrained than consumers in food webs, while animals show more constraints than plants in mutualistic networks. Third, phylogenetic constraints are stronger for the matching component than for the centrality component of network structure. These results can shed light on the contrasting evolutionary constraints shaping network structure across interaction types and species sets.
\end{abstract}

Keywords: food webs, plant-pollinator networks, plant-frugivore networks, phylogeny.

\section{Introduction}

In the last few years, ecologists have started incorporating phylogenetically based methods in ecological research (Webb et al. 2002; Helmus et al. 2007; Mouquet et al. 2012). Specifically, when studying ecological networks, they have concluded that network patterns have a phylogenetic signal and that, therefore, one cannot fully understand network architecture without considering the

* Corresponding author. Present address: Unit of Ecology and Evolution,
University of Fribourg, Chemin du Musee 10,1700 Fribourg, Switzerland;
e-mail: rudolf.rohr@unifr.ch.

Am. Nat. 2014. Vol. 184, pp. 556-564. (C) 2014 by The University of Chicago. 0003-0147/2014/18405-54531\$15.00. All rights reserved.

DOI: $10.1086 / 678234$ evolutionary history of constituent species (Cattin et al. 2004; Rossberg et al. 2006; Rezende et al. 2007a, 2007b, 2009; Bersier and Kehrli 2008; Rohr et al. 2010; Eklof et al. 2012; Mouquet et al. 2012; Naisbit et al. 2012). These studies have immediate implications for our understanding of network assembly (Cattin et al. 2004; Rossberg et al. 2006), the rate at which evolutionary history is lost as networks disassemble (Rezende et al. 2007a), and which ecological variables best correlate with network structure (Rezende et al. 2007b; Bersier and Kehrli 2008; Petchey et al. 2008; Rohr et al. 2010; Eklof et al. 2012; Naisbit et al. 2012). They also pave the road toward a predictive assessment of the networkwide consequences of biological invasions (Ives and Godfray 2006).

The above-described studies addressed either food webs (here referred to as antagonistic networks) or mutualistic networks, targeted different components of network structure (e.g., species degree or trophic similarity), and employed different methodologies (e.g., Bloomberg et al.'s $K$ or Mantel test). This has precluded an assessment of whether the amount of phylogenetic signal is similar across network types, species sets, and components of network structure. The aim of the present article is to provide a general framework to explore the relationship between phylogeny and network structure.

Our first step is to choose a model that can be applied to either directed networks (here represented by food webs) or bipartite networks (here represented by mutualistic webs). The model has to be versatile in capturing simultaneously the nested and modular structures of these networks, the two major structural properties found in mutualistic networks and food webs, respectively (Bascompte et al. 2003; Olesen et al. 2007; Fortuna et al. 2010; Thébault and Fontaine 2010).

We use the matching-centrality model that captures simultaneously the modular and nested structures by fitting at the same time the matching and centrality components of network architecture (Rohr et al. 2013). In this model, 
each species is characterized by two traits: one trait of matching and one trait of centrality. These traits quantify the position of the species in the network; the matching trait quantifies the assortative pattern and is thus able to capture the modular structure, while the centrality trait quantifies the variability in degree and thus captures the nested structure (fig. 1). The matching and centrality traits are not species' traits measured on organisms; they are inferred from the network itself. The power of the matching-centrality model is to fit simultaneously both components of network structure in both network types.

Intuitively, if species' traits explain network architecture, then they should be related to the matching and centrality traits. A typical example of a matching trait in a plantfrugivore network could be the size of the seed and the size of the bird's beak; these two traits have to match as much as possible to optimize the chance of a mutualistic interaction between that plant and that bird. We could also think of the length of a pollinator's proboscis, which has to match the length of the flower's corolla. A second example of a matching trait could be phenology. Indeed, the phenology of both species has to overlap to increase the probability of the two species to interact. Phenology could also be a centrality trait, since the longer the species is present in that location, the higher the number of its mutualistic partners and, in turn, the larger its centrality value. In the present article, we compare phylogenetic signal across network types and architecture components. Although the presence of phylogenetic constraints is not formal proof that matching and centrality traits are linked to species' traits, it is a good indication of this correspondence.

Our second step consists of fitting the matching-centrality model to a data set of 60 networks including food webs and mutualistic networks. These fits provide, independently for each network, the estimated traits of matching and centrality of all interacting species. Our final step consists of testing the presence and quantifying the strength of phylogenetic signal in the above-described network traits. This will allow us to determine in which type of network phylogenetic constraints are stronger and to compare these constraints between component types (matching and centrality) and species sets (animals, plants, prey, and predators).

\section{Material and Methods}

\section{Data Sets}

Our data set included 12 food webs, 19 plant-frugivore webs, and 29 plant-pollinator webs. The food webs were extracted from the database of Brose et al. (2005); specifically, we used here the networks for which the trophic interactions were determined from direct observation, as has been done by
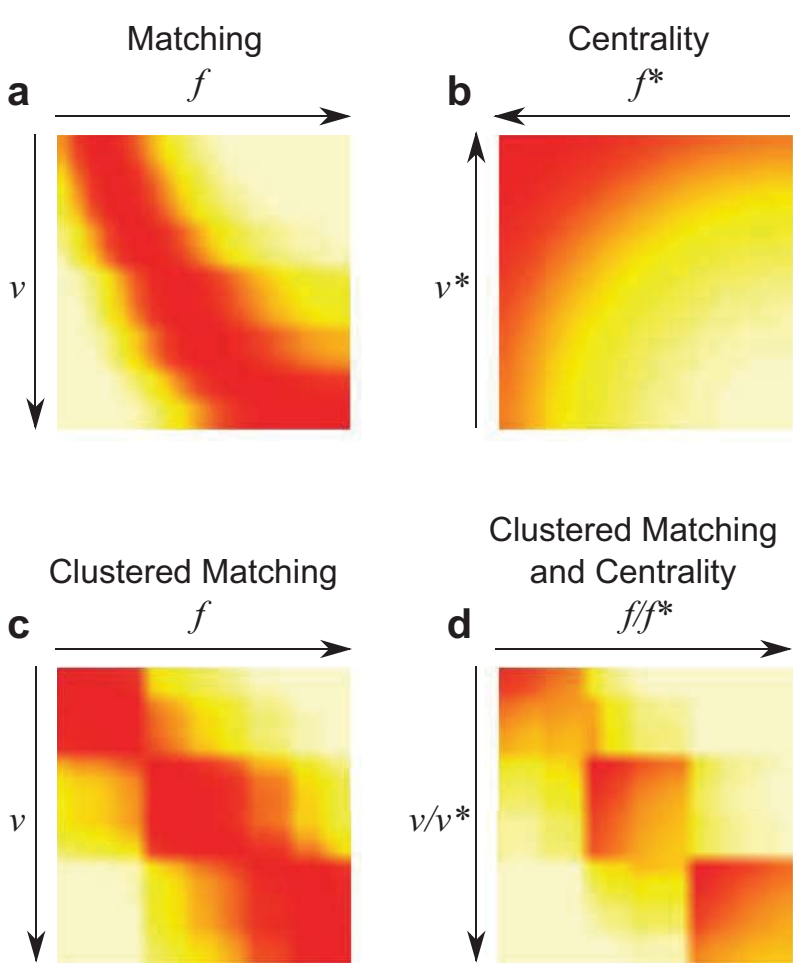

Figure 1: Sketch of network architectures inferred by the matchingcentrality model. Each panel represents the matrix of simulated linking probabilities for different conditions. Color goes from light yellow to dark red as the linking probability increases. In $a$ and $c$, the networks are driven only by the matching term, and the species are ordered according to their matching traits. While in $a$ the matching traits are sampled uniformly, they are clustered in three sets in $c$. In both panels, higher linking probabilities are located on the diagonal, and they are specifically clustered in three modules in $c$. In $b$, the network is driven only by the centrality term, and the species are ordered according to their centrality traits. Note that the linking probabilities are perfectly nested. In $d$, the network is driven by both matching and centrality traits, and the matching traits are clustered in three sets. The species are ordered according to cluster and within the cluster according to centrality traits. When both terms are present, the model is able to capture simultaneously the modular and nested structures of the networks.

Rohr et al. (2010) and Naisbit et al. (2011, 2012). Both the plant-frugivore and the plant-pollinator webs were extracted from the recently compiled data set of Rezende et al. (2007b). These networks are also available at http:// www.web-of-life.es and in the Dryad Digital Repository (http://dx.doi.org/10.5061/dryad.5m0m5; Rohr and Bascompte 2014).

For the food webs, we used taxonomy as a proxy for phylogeny. Taxonomic information was retrieved mainly from the Integrated Taxonomic Information System (http://www.itis.gov). Taxonomic trees were constructed according to 18 levels from species to kingdom. The dis- 
tance between two species is given as twice the number of taxonomic levels at which they differ. Further information can be found in Naisbit et al. (2012). For the mutualistic networks, we used the same phylogenies compiled by Rezende et al. (2007b), based on DNA sequences; plant phylogenies were compiled using Phylomatic, while insect phylogenies were based on the Tree of Life.

\section{Matching-Centrality Model}

Let $A_{i j}$ be the adjacency matrix of a web, that is, $A_{i j}=$ 1 if there is an interaction between species $i$ and $j$, and $A_{i j}=0$ otherwise. In mutualistic networks, $i$ represents plants while $j$ represents animals, and $A_{i j}=1$ if animal $j$ pollinates or disperses the seeds of plant $i$. In food webs, $i$ represents the species viewed as a resource while $j$ represents the species viewed as a consumer, and $A_{i j}=1$ if prey $i$ is eaten by predator $j$.

The aim of the matching-centrality model is to infer simultaneously the nested and modular structure of antagonistic and mutualistic networks (Rohr et al. 2013). The probability of establishing a link between species $i$ and $j$, denoted by $P\left(A_{i j}=1\right)$, is modeled as a function of their matching traits, denoted by $v_{i}$ and $f_{j}$, and their centrality traits, denoted by $v_{i}^{*}$ and $f_{j}^{*}$. Each plant, pollinator, prey, and predator is characterized by one trait of matching and one trait of centrality.

The model is implemented so that the closer the matching traits of two species, the higher the probability that they are linked. Similarly, the higher the centrality trait of a species, the higher the probability that this species establishes links. In other words, the matching traits quantify the assortative pattern, and the centrality traits quantify the species generality/specificity. The formula of the model is given by (Rohr et al. 2013):

$$
\operatorname{logit}\left(P\left(A_{i j}=1\right)\right)=\underbrace{-\lambda\left(v_{i}-f_{j}\right)^{2}}_{\text {matching term }}+\underbrace{\delta_{1} v_{i}^{*}+\delta_{2} f_{j}^{*}}_{\text {centrality term }},
$$

where $\lambda, \delta_{1}$, and $\delta_{2}$ are positive parameters providing the relative importance of matching, centrality for the resources or plants, and centrality for the consumers or animals, respectively. In the case of food webs, the denomination of these traits has a meaning: $v$ represents the vulnerability of prey, while $f$ represents the predator's foraging. As in the matching model (Rossberg et al. 2006), the foraging traits have to match as close as possible the vulnerability trait to maximize the probability of a trophic link. The same formula for the right side was first proposed by Rossberg et al. $(2010 a, 2013)$ when studying interaction strength in food webs and was used later in the context of the niche dimensionality in food webs (Rossberg et al. 2010b; Brännström et al. 2011). However, we derived our model within a more restricted framework than the more general one used by Rossberg et al. $(2010 a, 2013)$. This naturally results in constraints on our matching and centrality traits. Such constraints reduce the degrees of freedom, and therefore the traits are uniquely defined. We provide a complete mathematical derivation in the appendix (available online).

The nested structure (if this structure is present) is captured by the centrality traits, while modularity (if this structure is present) is embedded in the matching traits. Figure 1 represents four simulated matrices of linking probabilities generated by the matching-centrality model. In figure $1 b$, we assume that only the centrality term is present (i.e., $\lambda=0$ ), and the species are ordered according to their centrality traits. A nested structure clearly appears in the linking probabilities; thus, the model is perfectly tailored to fit highly nested networks. In figure $1 a$, the centrality term is equal to 0 (i.e., $\delta_{1}=0$ and $\delta_{2}=0$ ), and the matching traits are uniformly distributed. When species are ordered according to their matching traits, a diagonal structure clearly appears. Now, if we assume that the matching traits are aggregated in clusters, as we can see in figure $1 c$, the modular structure appears in the diagonal. Thus, the matching centrality model is very flexible and can infer networks whose architecture is a mixture between nestedness and modularity, as illustrated by figure $1 d$ (see also Lewinsohn et al. 2006). This is an important property for a candidate model to fit both food webs and mutualistic networks.

Different methods to quantify the phylogenetic signal in ecological networks already exist. For example, we can quantify the dietary or predatory similarity in food webs (Cattin et al. 2004; Bersier and Kehrli 2008; Naisbit et al. 2012) or the ecological similarity in mutualistic networks (Rezende et al. 2007b) and then use a Mantel correlation to test for phylogenetic signal. These approaches have advanced our knowledge of the phylogenetic constraints, but they are limited in the sense of looking only at a particular level (prey, predators, animals, or plants). Here, by inferring the traits of matching and centrality, we take a step forward and also look at the component of network architecture.

As each species is characterized by a matching and a centrality trait, the number of parameters to be estimated scales as twice the number of species. Moreover, the logit of the linking probability is not a linear function, as is normally the case. Thus, its inference is a complex task. The nonlinearity of the model implies that the traditional deterministic optimization algorithm for finding the maximum likelihood estimator may not work; a stochastic algorithm is more adequate. Thus, here we take advantage of a Bayesian framework and use a Monte Carlo Markov chain approach. The posterior distribution of the matching 
modularity of pollination networks. Proceedings of the National Academy of Sciences of the USA 104:19891-19896.

Petchey, O. L., A. P. Beckerman, J. O. Riede, and P. H. Warren. 2008. Size, foraging, and food web structure. Proceedings of the National Academy of Sciences of the USA 105:4191-4196.

Pires, M. M., P. I. Prado, and P. R. Guimarães Jr. 2011. Do food web models reproduce the structure of mutualistic networks? PLoS ONE 6:e27280.

Rezende, E. L., E. M. Albert, M. A. Fortuna, and J. Bascompte. 2009. Compartments in a marine food web associated with phylogeny, body mass, and habitat structure. Ecology Letters 12:779-788.

Rezende, E. L., P. Jordano, and J. Bascompte. 2007a. Effects of phenotypic complementarity and phylogeny on the nested structure of mutualistic networks. Oikos 116:1919-1929.

Rezende, E. L., J. E. Lavabre, P. R. Guimarães Jr., P. Jordano, and J. Bascompte. 2007b. Non-random coextinctions in phylogenetically structured mutualistic networks. Nature 448:925-928.

Rohr, R. P., and J. Bascompte. 2014. Data from: Components of phylogenetic signal in antagonistic and mutualistic networks. American Naturalist, Dryad Digital Repository, http://dx.doi.org /10.5061/dryad.5m0m5.

Rohr, R. P., R. E. Naisbit, C. Mazza, and L.-F. Bersier. 2013. Matchingcentrality decomposition and the forecasting of new links in networks. arXiv:1310.4633.

Rohr, R. P., H. Scherer, P. Kehrli, C. Mazza, and L. Bersier. 2010. Modeling food webs: exploring unexplained structure using latent traits. American Naturalist 173:170-177.
Rossberg, A. G. 2013. Food webs and biodiversity: foundations, models, data. Wiley, New York.

Rossberg, A. G., A. Brännström, and U. Dieckmann. 2010a. How trophic interaction strength depends on traits. Theoretical Ecology 3:13-24.

2010b. Food-web structure in low- and high-dimensional trophic niche spaces. Journal of the Royal Society Interface 53: 1735-1743.

Rossberg, A. G., H. Matsuda, T. Amemiya, and K. Itoh. 2006. Food webs: experts consuming families of experts. Journal of Theoretical Biology 241:552-563.

Stouffer, D., and J. Bascompte. 2011. Compartmentalization increases food-web persistence. Proceedings of the National Academy of Sciences of the USA 108:3648-3652.

Thébault, E., and C. Fontaine. 2010. Stability of ecological communities and the architecture of mutualistic and trophic networks. Science 329:853-856.

Thompson, J. N. 2006. Mutualistic webs of species. Science 312:372373.

Webb, C. O., D. D. Ackerly, M. A. McPeek, and M. J. Donoghue. 2002. Phylogenies and community ecology. Annual Review of Ecology and Systematics 33:475-505.
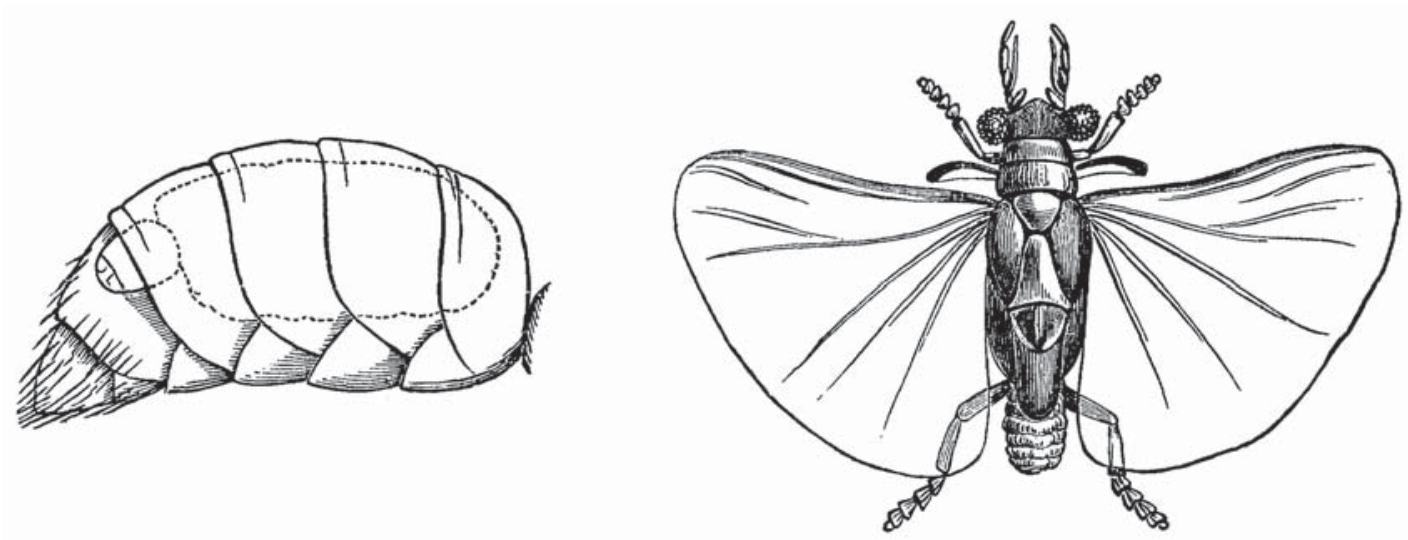

"On looking at my capture I saw a pale reddish-brown triangular mark on the bee's abdomen; this was the flattened head and thorax of a female Stylops [left]. ... On the last of April, when the Mezereon was in blossom, I caught the singular-looking male, Stylops Childreni Gray ..., which was as unlike its partner as possible." From "The Parasites of the Honey-Bee” by A. S. Packard Jr. (American Naturalist, 1868, 2:195-205). 

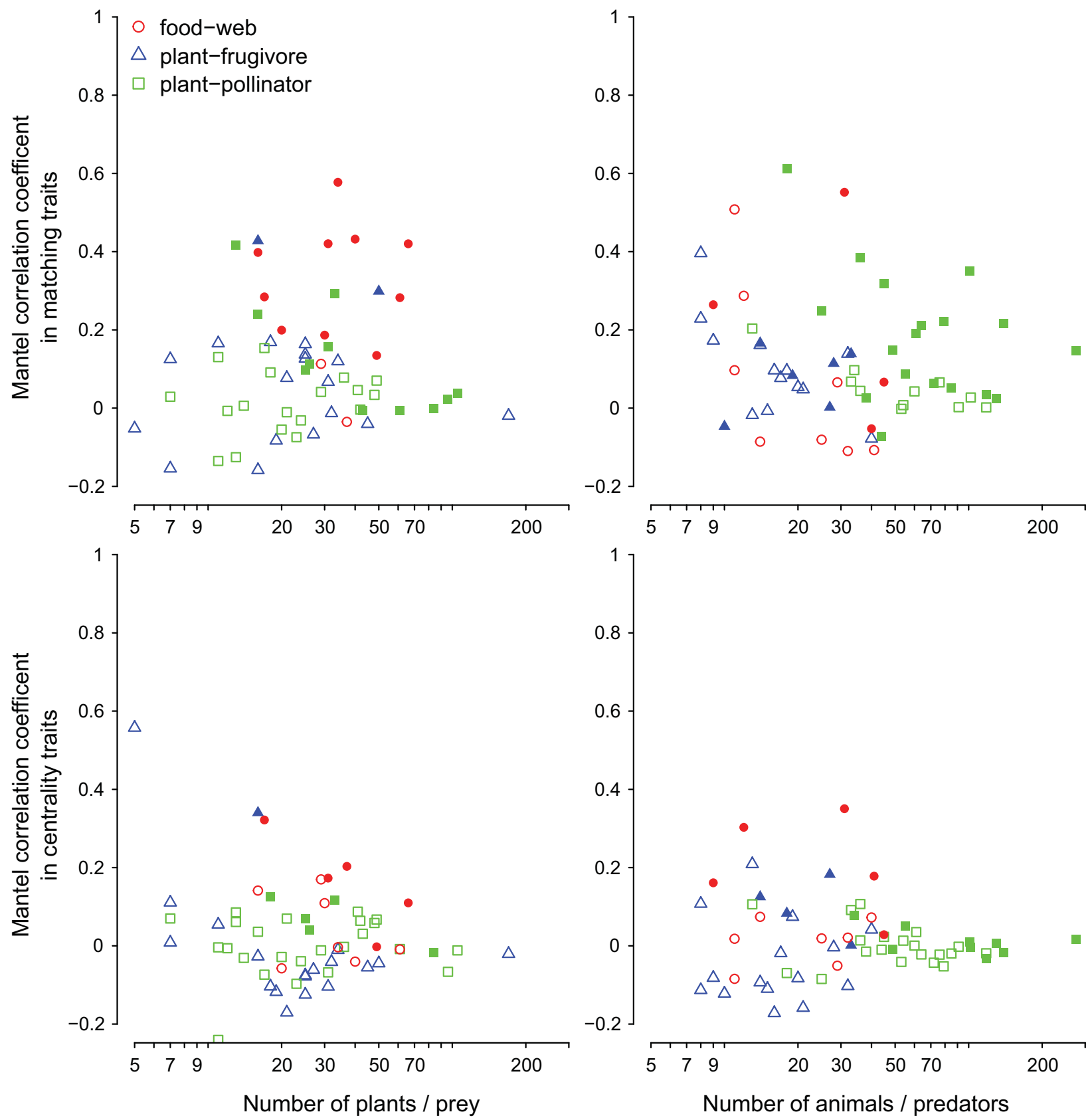

Figure 3: Strength of phylogenetic signal in the matching and centrality traits. For each type of web and species set, the strength of the phylogenetic signal found in the matching and centrality traits as function of the number of species are provided. Filled symbols represent a significant phylogenetic signal (at $\alpha=.05)$.

Strength of Phylogenetic Signal. We quantified the strength of the phylogenetic signal using the Mantel correlation coefficient (Cattin et al. 2004; Rezende et al. 2007b; Rohr et al. 2010; Naisbit et al. 2012). As distance matrices, we used the pairwise Euclidian distance matrix in the matching and centrality traits (Rohr et al. 2010) and the matrix of pairwise distances in the phylogenetic tree (Cattin et al. 2004; Rezende et al. 2007b; Naisbit et al. 2012). The Mantel correlation coefficient also has the advantage of having a low sensitivity to the branch length of the phylogenetic tree, making the comparison between food webs and mutualistic networks reasonable. 


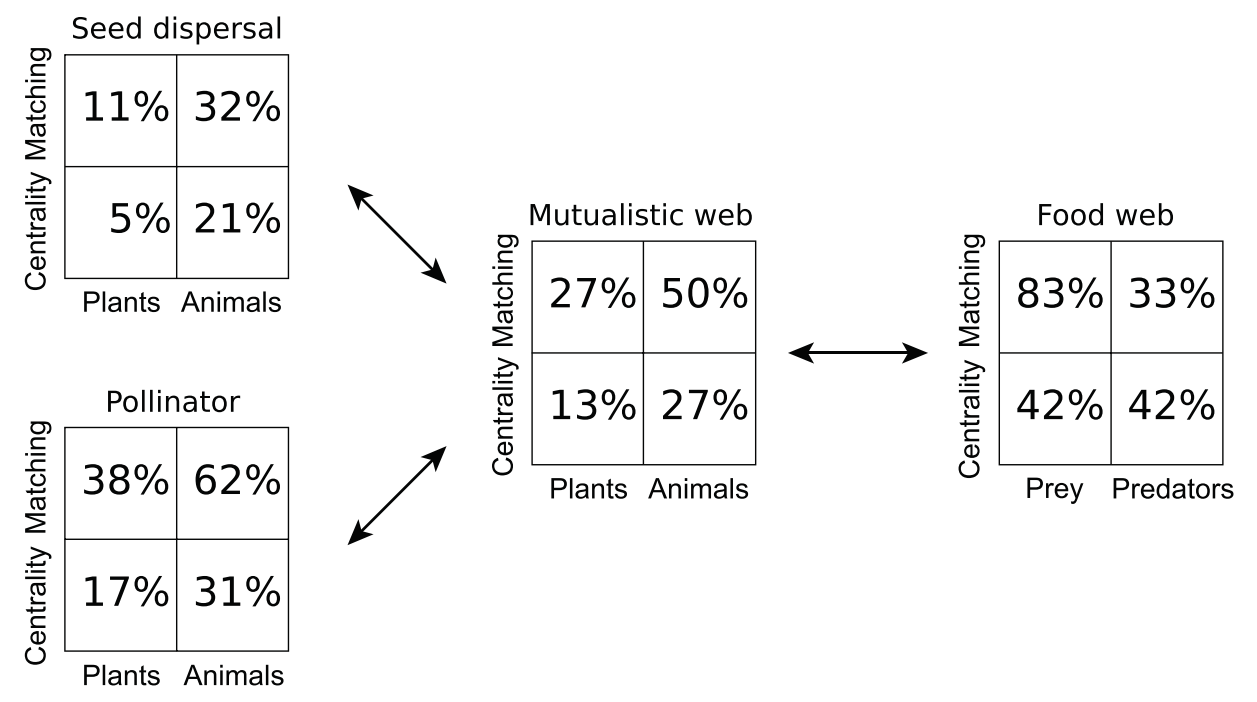

Figure 4: Presence of phylogenetic signal in the matching and centrality traits. For each type of web and species set, the percentage of significant $P$ values (at $\alpha=.05$ ) found in the matching and centrality traits are provided.

\section{Results}

\section{Matching versus Centrality}

Figure 2 shows the relative contribution of the centrality and matching terms. For each web, we represent the relative contribution of the prey/plant centrality term over the matching term $\left(\log \left(\delta_{1} / \lambda\right)\right)$ versus the relative contribution of the predator/animals centrality term over the matching terms $\left(\log \left(\delta_{2} / \lambda\right)\right)$. For networks located at the top right, the overall contribution of the centrality term over the matching term is more important in explaining the architecture of the network, and vice versa for webs located at the bottom left. Food webs are mainly located at the bottom left, while mutualistic networks are mainly located at the top right $(t$ test: $P \leq .001$ for both $\log \left(\delta_{1} / \lambda\right)$ and $\log \left(\delta_{2} / \lambda\right)$ axes). This proves that, in general, the matching term (i.e., the modular structure) plays a more important role in describing food web structure, while mutualistic networks are best described by the centrality component (i.e., the nested structure; see also Thébault and Fontaine 2010). However, there is an overlap area in the middle.

\section{Phylogenetic Signal}

For each web, we measured the strength and tested the presence of phylogenetic signal in the fitted matching and centrality traits. Figure 3 depicts the strength (measured by the Mantel correlation coefficient) and the significance (based on the $K$ statistic) of the phylogenetic signal in the matching and centrality traits for each network type.

Regarding the frequency of phylogenetic signal, we found a significant phylogenetic signal (at $\alpha=.05$ ) in both the matching and the centrality traits of the plants, animals, prey, and predators in all food webs and in $63 \%$ of the mutualistic webs. This difference is significant, with $P=.029$. If we split the mutualistic networks into plantpollinator and plant-frugivore webs, then the percentages of networks with a significant phylogenetic signal are 79\% (for plant-pollinators) and 37\% (for plant-frugivores). The difference between food webs and plant-pollinators is not significant $(P=.223)$, but the differences between food webs and plant-pollinators on the one hand and plantfrugivores on the other are significant $(P=.002$ and .008 , respectively). Thus, plant-frugivore webs are the ones accounting for the overall fewer cases of significant phylogenetic signal in mutualistic networks.

Figure 4 provides a summary of the presence of phylogenetic signal according to type of web, species, and traits. Overall, we found a significant difference between matching and centrality traits $(P=.004)$. Specifically, phylogenetic signal is more often present in the matching traits than in the centrality traits. We found a second asymmetry in the matching traits between food webs and mutualistic networks. For the former the rate of significant phylogenetic signal is higher for prey than for predators $(P=.007)$, while for mutualistic networks the rate of significance is higher for animals than for plants $(P=.011)$.

Turning to the strength of phylogenetic signal (fig. 5; tables A1, A2; tables A1, A2 are available online), we found the same general pattern as for its presence. This is not surprising, as we can expect that the higher the phylogenetic constraints, the easier it is to detect them. In general, we found a higher correlation in matching traits than in centrality traits (table A1). Thus, traits determining the 


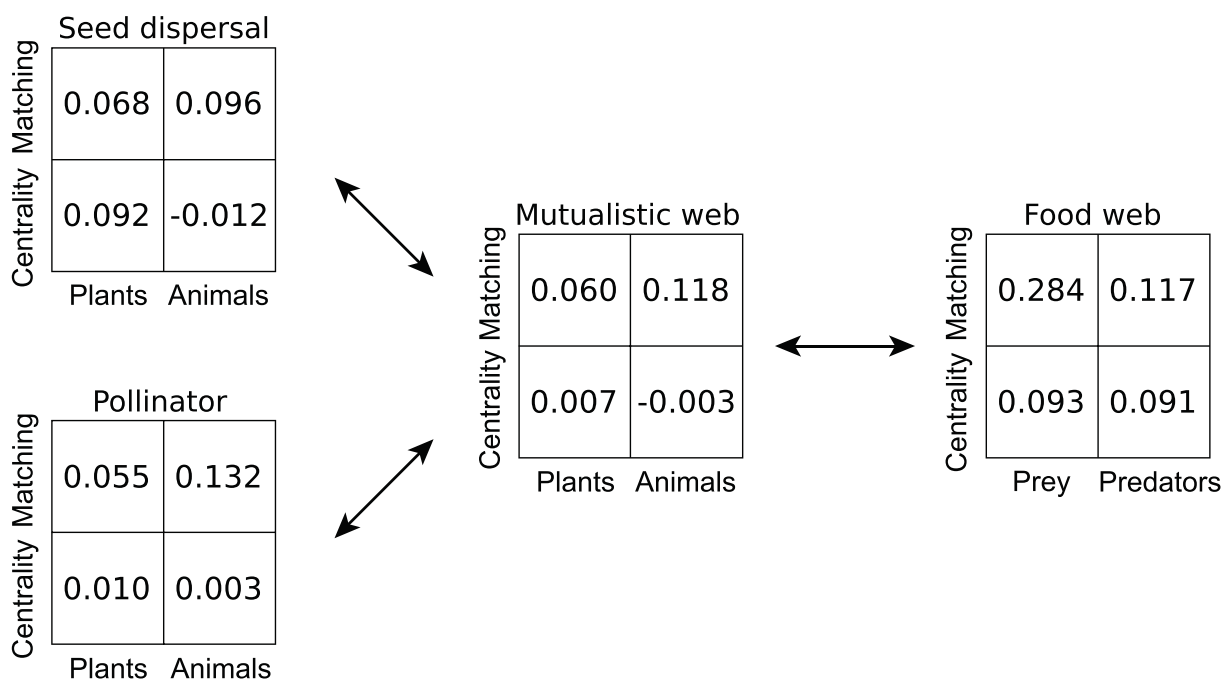

Figure 5: Mean phylogenetic strength in the matching and centrality traits. For each type of web and species set, the mean phylogenetic strength found in the matching and centrality traits are provided.

modular component are more phylogenetically constrained than those quantifying the nested component in all types of networks.

In food webs, phylogenetic constraints are stronger in the matching traits of the prey than in the other traits/ species sets, while in mutualistic networks the matching traits of animals exhibit the strongest phylogenetic constraints (table A1). Regarding the difference between food webs and mutualistic networks, phylogenetic constraints are always stronger in the former (table A2).

However, the difference in the frequency of significant phylogenetic signal between plant-pollinator and plantfrugivore networks does not hold for the strength of such phylogenetic signal. As plant-frugivore networks are smaller that plant-pollinator ones in our data set, a power issue cannot be excluded. Thus, this specific result on the rate of significance should be taken with caution.

\section{Discussion}

As a preliminary step to incorporate phylogenetic structure, in this article we have used the matching-centrality model to characterize two components of network structure. Our results have shown that the matching component is relatively stronger than the centrality component in food webs, while the opposite occurs in mutualistic networks. This result is not so surprising, as it has already been pointed out that food webs are more modular than nested, while mutualistic networks are more nested than modular (Fortuna et al. 2010; Thébault and Fontaine 2010). This is an indirect confirmation that our matching and centrality traits are quantifying species position in the modular and the nested component, respectively. It has been shown that this difference in component of network architecture has dynamic consequences. Thus, modular food webs are known to be more persistent (Stouffer and Bascompte 2011), while nestedness promotes biodiversity in mutualistic networks (Bastolla et al. 2009; Thébault and Fontaine 2010).

This difference in network architecture is also compatible with coevolutionary theory (Thompson 2006; Guimarães et al. 2011; Nuismer et al. 2013). Specifically, antagonistic coevolution between prey and predators may lead toward an arms-race escalation, generating modules of species sharing some defenses and counterdefenses. Mutualistic interactions, on the other hand, may lead to nested networks through the combination of complementarity and convergence of traits (Thompson 2006).

Once we quantified the two components of network structure, we proceeded by adding the phylogenies. Our results show three contrasting patterns regarding differences in phylogenetic signal in ecological networks. First, phylogenetic constraints are stronger in antagonistic networks than in mutualistic networks. Second, the matching component exhibits a stronger phylogenetic signal than the centrality component across network types. Third, prey are more phylogenetically constrained than predators in food webs, while animals are more constrained than plants in mutualistic networks.

Regarding our first result—namely, a stronger effect of evolutionary history on food webs than on mutualistic networks - this can be explained by the different taxonomic heterogeneity between these two types of networks. Thus, food webs may encompass organisms quite different 
across the evolutionary tree, such as higher plants, arthropods, and birds. In turn, mutualistic networks tend to contain a smaller diversity of taxonomic groups, such as insects in plant-pollinator networks and frugivorous birds in plant-dispersal networks.

Regarding the second result-namely, a stronger phylogenetic constraint in the matching than in the centrality component-we could presume coevolution as a potential explanation for this difference (Thompson 2006; Guimarães et al. 2011; Nuismer et al. 2013). This, however, needs more rigorous testing, and future studies simulating coevolution, as opposed to simply evolution, in these networks could shed light on this matter.

Finally, our last finding is that prey are more phylogenetically constrained than predators in food webs, while animals are more constrained than plants in mutualistic networks. Bersier and Kehrli (2008) have pointed out this asymmetry for food webs, while Rezende et al. (2007b) independently found a similar asymmetry in mutualistic networks. Surprisingly, these two asymmetries go in opposite directions. This is so because one usually considers animals to be consumers (predators) and plants to be resources (prey) in mutualistic networks (e.g., see Pires et al. 2011), so if prey are more phylogenetically constrained in food webs, we could expect the same for plants in mutualistic networks. This result suggests that mutualistic networks, despite also being a representation of consumerresource webs, are quite different from traditional food webs. While in the latter the interaction is $+/-$, with one species gaining and the other losing from the interaction, in mutualistic networks the interaction is $+/+$. This important difference could explain the contrasting patterns in the magnitude of phylogenetic signal. Mutualistic networks are a unique type of ecological network.

Studies of ecological networks have benefited in the last few years from the incorporation of phylogenetically based methods. This has provided a link between current network architecture and evolutionary history. Our current results may be useful in expanding these previous fragmented results by building a unified framework where these sorts of questions can be addressed in a comparative way across different network types.

\section{Acknowledgments}

R.P.R. and J.B. designed the research, R.P.R. performed the simulations and analysis, and R.P.R. and J.B. wrote the manuscript. We thank L.-F. Bersier, P. Jordano, P. Kehrli, and R. Nasibit for their input. This work was funded by the FP7-REGPOT-2010-1 program (project 264125 EcoGens) and a European Research Council advanced grant.

\section{Literature Cited}

Bascompte, J., P. Jordano, C. J. Melián, and J. M. Olesen. 2003. The nested assembly of plant-animal mutualistic networks. Proceedings of the National Academy of Sciences of the USA 100:9383-9387.

Bastolla, U., M. Fortuna, A. Pascual-Garcia, A. Ferrera, B. Luque, and J. Bascompte. 2009. The architecture of mutualsitic networks minimizes competition and increases biodiversity. Nature 458: 1018-1021.

Bersier, L. F., and P. Kehrli. 2008. The signature of phylogenetic constraints on food-web structure. Ecological Complexity 5:132139.

Blomberg, S. P., T. Garland, and A. R. Ives. 2003. Testing for phylogenetic signal in comparative data: behavioral traits are more labile. Evolution 57:717-745.

Brännström, A., L. Carlsson, and A. G. Rossberg. 2011. Rigorous conditions for food-web intervality in high-dimensional trophic niche spaces. Journal of Mathematical Biology 63:572-592.

Brose, U., L. Cushing, E. L. Berlow, T. Jonsson, C. Banasek-Richter, L.-F. Bersier, J. L. Blanchard, et al. 2005. Body sizes of consumers and their resources. Ecology 86:2545.

Cattin, M. F., L. F. Bersier, C. Banasek-Richter, R. Baltensperger, and J. P. Gabriel. 2004. Phylogenetic constraints and adaptation explain food-web structure. Nature 427:835-839.

Eklof, A., M. R. Helmus, M. Moore, and S. Allesina. 2012. Relevance of evolutionary history for food web structure. Proceedings of the Royal Society B: Biological Sicences 279:1588-1596.

Fortuna, M. A., D. B. Stouffer, J. M. Olesen, P. Jordano, D. Mouillot, B. R. Krasnov, R. Poulin, and J. Bascompte. 2010. Nestedness versus modularity in ecological networks: two sides of the same coin? Journal of Animal Ecology 79:811-817.

Gilks, W., S. Richardson, and D. Spiegelhater. 1996. Markov Chain Monte Carlo in practice. Chapman \& Hall, London.

Grafen, A. 1989. The phylogenetic regression. Philosophical Transactions of the Royal Society B: Biological Sciences 326:119-157.

Guimarães, P. R., Jr., P. Jordano, and J. N. Thompson. 2011. Evolution and coevolution in mutualistic networks. Ecology Letters 14:877885.

Helmus, M. R., K. Savage, M. W. Diebel, J. T. Maxted, and A. R. Ives. 2007. Separating the determinants of phylogenetic community structure. Ecology Letters 10:917-925.

Ives, A. R., and H. C. J. Godfray. 2006. Phylogenetic analysis of trophic associations. American Naturalist 168:E1-E14.

Lewinsohn, T. M., P. I. Prado, P. Jordano, J. Bascompte, and J. M. Olesen. 2006. Structure in plant-animal interaction assemblages. Oikos 113:174-184.

Mouquet, N., V. Devictor, C. N. Meynard, F. Munoz, L.-F. Bersier, J. Chave, P. Couteron, et al. 2012. Ecophylogenetics: advances and perspectives. Biological Reviews 87:769-785.

Naisbit, R. E., P. Kehrli, R. P. Rohr, and L.-F. Bersier. 2011. Phylogenetic signal in predator-prey body-size relationships. Ecology 92: 2183-2189.

Naisbit, R. E., R. P. Rohr, A. G. Rossberg, P. Kehrli, and L.-F. Bersier. 2012. Phylogeny versus body size as determinants of food web structure. Proceedings of the Royal Society B: Biological Sciences 279:3291-3297.

Nuismer, S., P. Jordano, and J. Bascompte. 2013. Coevolution and the architecture of mutualistic networks. Evolution 67:338-354.

Olesen, J. M., J. Bascompte, Y. L. Dupont, and P. Jordano. 2007. The 
modularity of pollination networks. Proceedings of the National Academy of Sciences of the USA 104:19891-19896.

Petchey, O. L., A. P. Beckerman, J. O. Riede, and P. H. Warren. 2008. Size, foraging, and food web structure. Proceedings of the National Academy of Sciences of the USA 105:4191-4196.

Pires, M. M., P. I. Prado, and P. R. Guimarães Jr. 2011. Do food web models reproduce the structure of mutualistic networks? PLoS ONE 6:e27280.

Rezende, E. L., E. M. Albert, M. A. Fortuna, and J. Bascompte. 2009. Compartments in a marine food web associated with phylogeny, body mass, and habitat structure. Ecology Letters 12:779-788.

Rezende, E. L., P. Jordano, and J. Bascompte. 2007a. Effects of phenotypic complementarity and phylogeny on the nested structure of mutualistic networks. Oikos 116:1919-1929.

Rezende, E. L., J. E. Lavabre, P. R. Guimarães Jr., P. Jordano, and J. Bascompte. 2007b. Non-random coextinctions in phylogenetically structured mutualistic networks. Nature 448:925-928.

Rohr, R. P., and J. Bascompte. 2014. Data from: Components of phylogenetic signal in antagonistic and mutualistic networks. American Naturalist, Dryad Digital Repository, http://dx.doi.org /10.5061/dryad.5m0m5.

Rohr, R. P., R. E. Naisbit, C. Mazza, and L.-F. Bersier. 2013. Matchingcentrality decomposition and the forecasting of new links in networks. arXiv:1310.4633.

Rohr, R. P., H. Scherer, P. Kehrli, C. Mazza, and L. Bersier. 2010. Modeling food webs: exploring unexplained structure using latent traits. American Naturalist 173:170-177.
Rossberg, A. G. 2013. Food webs and biodiversity: foundations, models, data. Wiley, New York.

Rossberg, A. G., A. Brännström, and U. Dieckmann. 2010a. How trophic interaction strength depends on traits. Theoretical Ecology 3:13-24.

2010b. Food-web structure in low- and high-dimensional trophic niche spaces. Journal of the Royal Society Interface 53: 1735-1743.

Rossberg, A. G., H. Matsuda, T. Amemiya, and K. Itoh. 2006. Food webs: experts consuming families of experts. Journal of Theoretical Biology 241:552-563.

Stouffer, D., and J. Bascompte. 2011. Compartmentalization increases food-web persistence. Proceedings of the National Academy of Sciences of the USA 108:3648-3652.

Thébault, E., and C. Fontaine. 2010. Stability of ecological communities and the architecture of mutualistic and trophic networks. Science 329:853-856.

Thompson, J. N. 2006. Mutualistic webs of species. Science 312:372373.

Webb, C. O., D. D. Ackerly, M. A. McPeek, and M. J. Donoghue. 2002. Phylogenies and community ecology. Annual Review of Ecology and Systematics 33:475-505.
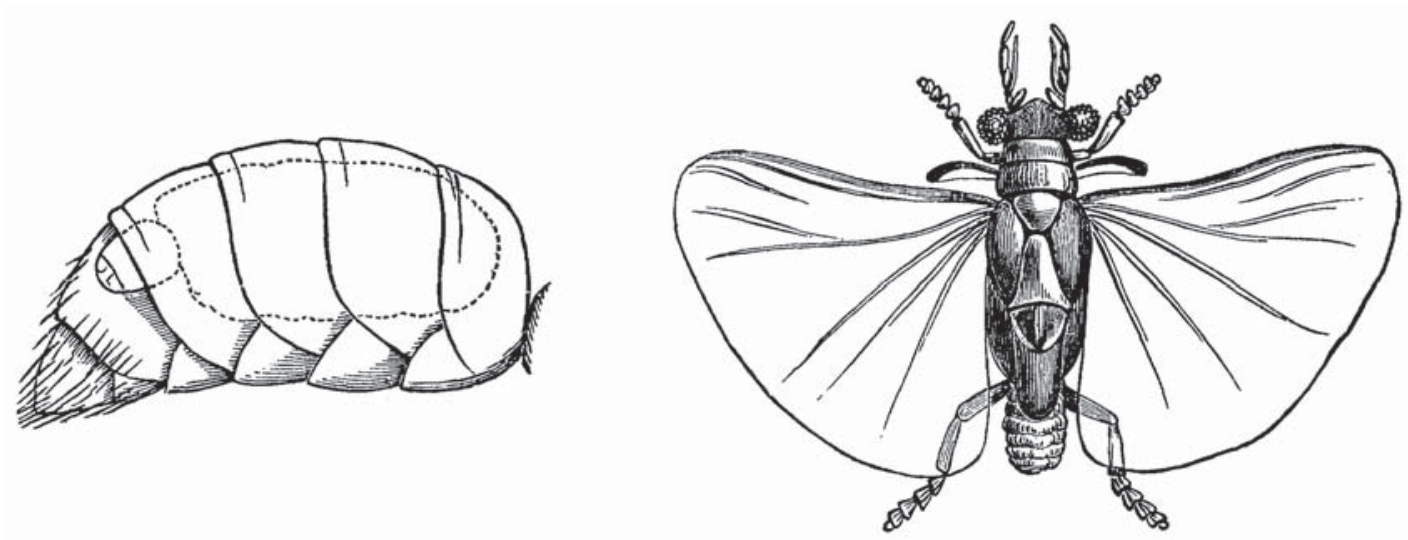

"On looking at my capture I saw a pale reddish-brown triangular mark on the bee's abdomen; this was the flattened head and thorax of a female Stylops [left]. ... On the last of April, when the Mezereon was in blossom, I caught the singular-looking male, Stylops Childreni Gray ..., which was as unlike its partner as possible." From "The Parasites of the Honey-Bee” by A. S. Packard Jr. (American Naturalist, 1868, 2:195-205). 